\title{
Maintenance of Stroke Care Quality amid the Coronavirus Disease 2019 Outbreak in Taiwan
}

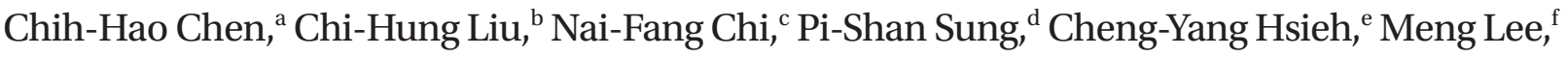 \\ Sung-Chun Tang, ${ }^{a}$ Jiann-Shing Jeng, ${ }^{a}$ on Behalf of the Taiwan Stroke Society Investigators \\ ${ }^{a}$ Department of Neurology, National Taiwan University Hospital, Taipei, Taiwan \\ bepartment of Neurology, Linkou Chang Gung Memorial Hospital, Taoyuan, Taiwan \\ 'Department of Neurology, Taipei Veterans General Hospital, Taipei, Taiwan \\ ${ }^{d}$ Department of Neurology, National Cheng Kung University Hospital, College of Medicine, National Cheng Kung University, Tainan, Taiwan \\ 'Department of Neurology, Tainan Sin Lau Hospital, Tainan, Taiwan \\ fDepartment of Neurology, Chiayi Chang Gung Memorial Hospital, Chiayi, Taiwan
}

\section{Dear Sir:}

It is well known that the implementation of evidence-based stroke care guidelines can effectively improve outcomes and prevent recurrence in patients with stroke.' In 2010, Taiwan implemented a nationwide collaborative model called the Breakthrough Series (BTS)-Stroke activity, adapted from the Get With The Guideline-Stroke program; this significantly improved outcomes on quality measures of acute ischemic stroke (AIS) care. ${ }^{2}$

During the coronavirus disease 2019 (COVID-19) pandemic, routine care of stroke may be compromised because of realloca- tion of medical resources. In Taiwan, the first confirmed COVID-19 case was reported on January 21, 2020. Because of the Taiwanese government's aggressive containment efforts, ${ }^{3}$ the cumulative number of COVID-19 cases, as of May 2020, was as low as 442 . Whether the number of daily admissions and quality metrics for stroke care changed during the COVID-19 pandemic period warrants investigation.

We retrospectively analyzed registry-based data from 18 hospitals in Taiwan, including seven medical centers and 11 community hospitals. The 18 hospitals were distributed in Taiwan's different administrative districts and contained $>65 \%$ of the total
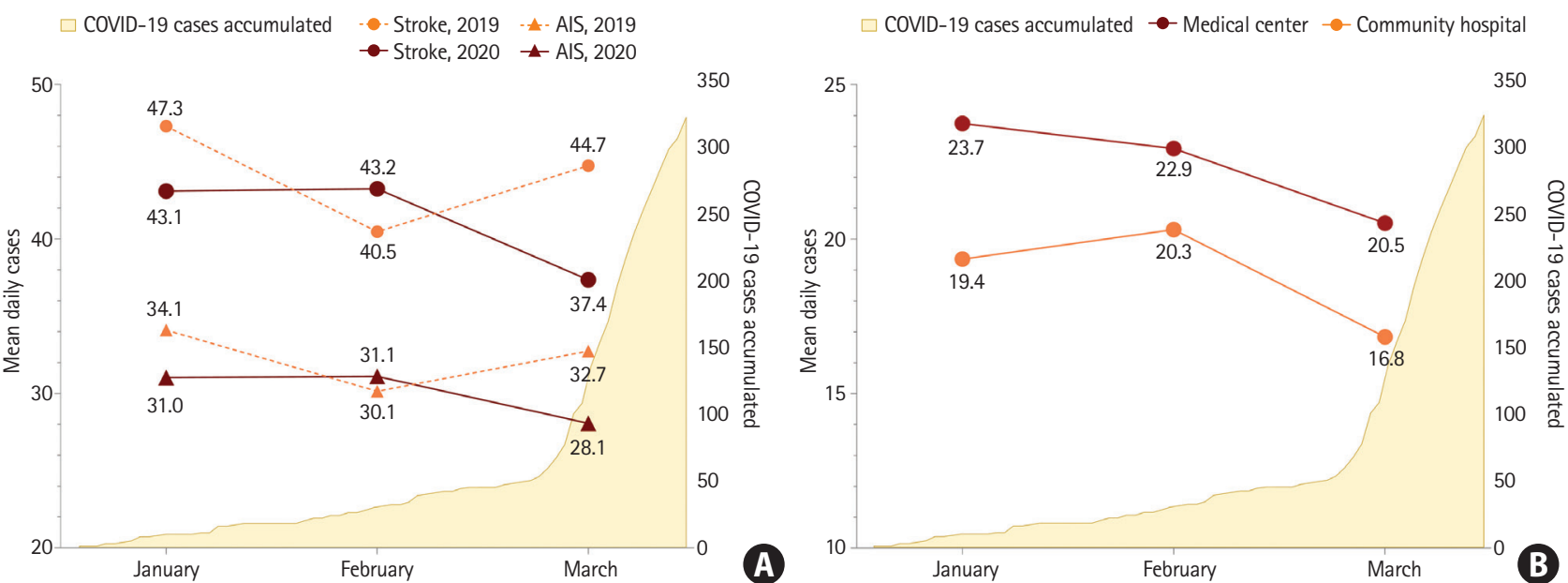

Figure 1. Trends of mean daily admissions of stroke between the first quarters of 2019 and 2020. (A) The rates of stroke and acute ischemic stroke (AIS) admissions decreased substantially over the first 3 months of 2020 ( $P<0.001$ and $P=0.03$, respectively), but not in $2019(P=0.13$ and $P=0.35$, respectively). (B) The decrease of stroke admission rate in 2020 were consistently found in medical centers $(P=0.007)$ and community hospitals $(P=0.02)$. COVID-19, coronavirus disease 2019 .

This is an Open Access article distributed under the terms of the Creative Commons Attribution Non-Commercial License (http://creativecommons.org/licenses/by-nc/4.0/) which permits unrestricted non-commercial use, distribution, and reproduction in any medium, provided the original work is properly cited. 
population (Supplementary Table 1). All the hospitals had participated in the BTS-Stroke activity. ${ }^{2}$ The performance measures and safety indicators were modified from the original BTSStroke quality metrics established in 2010 (Supplementary Table 2) and were reviewed monthly. Individual patient-level information was de-identified before analysis. Number of monthly admissions of stroke (including ischemic and hemorrhagic stroke) and 15 quality-of-care metrics were compared between the main outbreak (March 2020), early outbreak (January and February 2020), and control (January to March 2019) phases, respectively. Since the BTS-Stroke activity mainly focused on the AIS-related quality metrics, number of AIS admission were further recorded. Detailed methods and statistical analyses are presented in the Supplementary methods.

As the cumulative number of COVID-19 cases increased, there was a significant decrease in mean daily stroke admissions in the first quarter of $2020(\beta=-0.07, P<0.001)$, which was not ob- served in $2019(\beta=-0.03, P=0.13)$ (Figure 1A). Similar trends were observed in medical centers ( $\beta=-0.07, P=0.007)$ and community hospitals ( $\beta=-0.07, P=0.02$ ) (Figure $1 \mathrm{~B}$ ).

The comparison between the first quarter of 2019 and 2020 was presented in Table 1. The number of daily stroke admission were decreased in 2020 compared with 2019 (41.2 vs. 44.3; incidence rate ratio [IRR], $0.93 ; P=0.001)$ as well as AIS admission ( 29.9 vs. $32.6 ; I R R, 0.93 ; P=0.001$ ). The quality metrics were generally comparable, and several metrics of intravenous thrombolysis, endovascular thrombectomy, early and discharge antithrombotic use, and rehabilitation evaluation even improved in 2020.

Table 2 presents the aggregated data of stroke admissions and quality metrics across different study periods. During the main outbreak phase, the number of daily stroke admission was 37.4, which was an absolute decrease of 13\% compared to the early outbreak phase (43.2; IRR, 0.87; $P<0.001)$ and an absolute decrease of $16 \%$ compared to the control phase $(44.3 ;$ IRR, 0.84 ;

Table 1. Comparison of the stroke admissions and quality metrics between the first quarter of 2020 and 2019

\begin{tabular}{|c|c|c|c|c|}
\hline Study periods & Jan to Mar 2020 & Jan to Mar 2019 & Effect size, OR $(95 \% \mathrm{Cl})^{*}$ & $P$ \\
\hline \multicolumn{5}{|c|}{ Mean daily admissions, total (day average) } \\
\hline All stroke & $3,748(41.19)$ & $3,986(44.29)$ & $0.93(0.89-0.97)$ & 0.001 \\
\hline AIS & $2,734(30.04)$ & $2,916(32.40)$ & $0.93(0.88-0.98)$ & 0.001 \\
\hline AIS, medical centers & $1,464(16.09)$ & $1,565(17.39)$ & $0.93(0.86-0.99)$ & 0.03 \\
\hline AIS, community hospitals & $1,270(13.96)$ & $1,351(15.01)$ & $0.93(0.86-1.00)$ & 0.06 \\
\hline Early arrival $(<2 \mathrm{hr})$ of AIS & $18.1 \%(496 / 2,734)$ & $14.6 \%(427 / 2,916)$ & $1.29(1.12-1.49)$ & $<0.001$ \\
\hline \multicolumn{5}{|l|}{ Stroke severity by NIHSS score } \\
\hline$<4 / 4-10 / 11-20 />20$ & $1,060 / 874 / 424 / 282$ & $1,237 / 972 / 421 / 277$ & & 0.11 \\
\hline Mild stroke (NIHSS <4) & $40.2 \%(1,060 / 2,640)$ & $42.6 \%(1,237 / 2,907)$ & $0.91(0.81-1.01)$ & 0.07 \\
\hline \multicolumn{5}{|c|}{ Quality metrics, $\%$ (numerator/denominator) } \\
\hline Door-to-CT $\leq 25 \mathrm{~min}$ & $82.3 \%(408 / 496)$ & $83.6 \%(357 / 427)$ & $0.91(0.65-1.28)$ & 0.59 \\
\hline IV-tPA for early arrival & $96.6 \%(198 / 205)$ & $97.4 \%(151 / 155)$ & $0.79(0.24-2.59)$ & 0.69 \\
\hline IV-tPA percentage & $8.8 \%(238 / 2,696)$ & $6.3 \%(183 / 2,900)$ & $1.44(1.18-1.76)$ & $<0.001$ \\
\hline Door-to-needle $\leq 60 \mathrm{~min}$ & $66.4 \%(148 / 223)$ & $66.9 \%(119 / 178)$ & $0.98(0.65-1.49)$ & 0.92 \\
\hline Symptomatic ICH after IV-tPA & $3.8 \%(9 / 237)$ & $3.3 \%(6 / 182)$ & $1.13(0.41-3.13)$ & 0.82 \\
\hline EVT percentage & $8.0 \%(216 / 2,692)$ & $5.4 \%(158 / 2,912)$ & $1.46(1.18-1.81)$ & $<0.001$ \\
\hline Symptomatic ICH after EVT & $10.2 \%(22 / 216)$ & $7.6 \%(12 / 158)$ & $1.36(0.66-2.80)$ & 0.41 \\
\hline Dysphagia screening & $96.6 \%(2,091 / 2,171)$ & $95.7 \%(2,332 / 2,438)$ & $1.19(0.88-1.59)$ & 0.26 \\
\hline Early antithrombotics use & $98.6 \%(2,245 / 2,277)$ & $97.6 \%(2,504 / 2,566)$ & $1.72(1.12-2.65)$ & 0.01 \\
\hline Anticoagulants for $\mathrm{AF}$ & $92.6 \%(349 / 377)$ & $89.5 \%(323 / 361)$ & $1.46(0.88-2.43)$ & 0.14 \\
\hline Lipid-lowering drugs use & $94.3 \%(1,183 / 1,255)$ & $93.9 \%(1,289 / 1,373)$ & $1.07(0.77-1.48)$ & 0.68 \\
\hline Antithrombotics use at discharge & $97.0 \%(2,181 / 2,248)$ & $96.0 \%(2,394 / 2,495)$ & $1.37(1.00-1.87)$ & 0.049 \\
\hline Rehabilitation evaluation & $91.1 \%(2,044 / 2,243)$ & $87.6 \%(2,190 / 2,501)$ & $1.46(1.21-1.76)$ & $<0.001$ \\
\hline Stroke education & $97.3 \%(2,116 / 2,174)$ & $96.5 \%(2,330 / 2,414)$ & $1.31(0.94-1.84)$ & 0.12 \\
\hline 30-day mortality & $5.6 \%(129 / 2,318)$ & $6.8 \%(174 / 2,559)$ & $0.81(0.64-1.02)$ & 0.08 \\
\hline
\end{tabular}

$\mathrm{OR}$, odds ratio; $\mathrm{Cl}$, confidence interval; AIS, acute ischemic stroke; NIHSS, National Institutes of Health Stroke Scale; CT, computed tomography; IV-tPA, intravenous tissue plasminogen activator; ICH, intracerebral hemorrhage; $E V T$, endovascular thrombectomy; $A F$, atrial fibrillation.

*For rate data (case per day), the effect size was incidence rate ratio. 


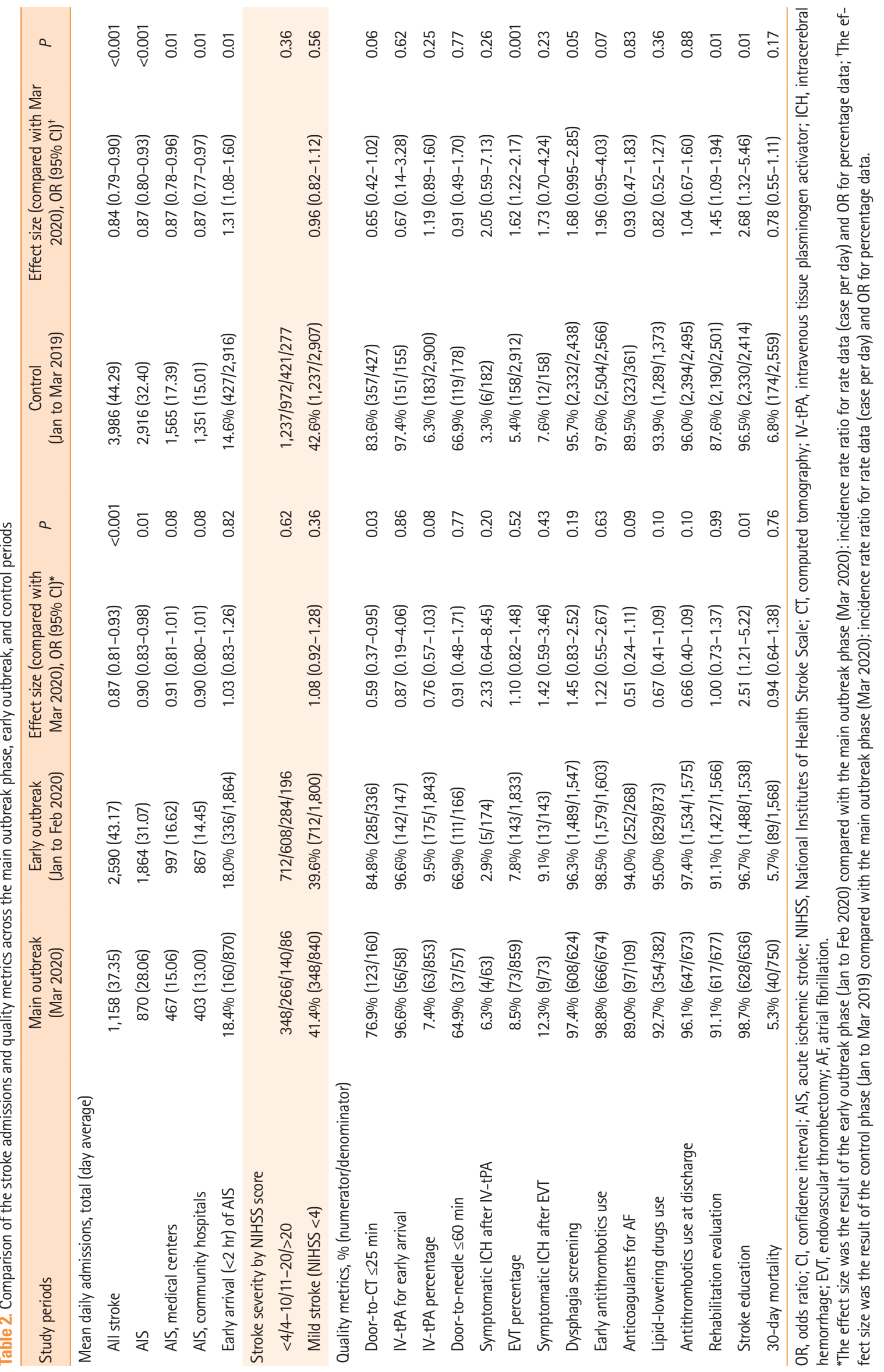


$P<0.001)$. Compared with the early outbreak phase, quality metrics were largely comparable except for fewer patients having a door-to-computed tomography time $\leq 25$ minutes in the main outbreak phase ( $76.9 \%$ vs. $84.8 \%$; odds ratio [OR], 0.59; $P=0.03)$. However, when compared to the control phase, the proportion of patients who arrived within 2 hours from stroke onset ( $18.4 \%$ vs. $14.6 \% ; O R, 1.31 ; P=0.01)$ and those who received endovascular thrombectomy (8.5\% vs. $5.4 \%$; OR, 1.62; $P=0.001)$ increased in the main outbreak phase. The quality metrics of rehabilitation evaluation and stroke education also improved.

We found that most stroke quality measures during the current study periods considerably improved compared to the initial BTS-Stroke activity implementation period of 2010 to $2011 .{ }^{2}$ More importantly, the overall quality of acute stroke care was well-maintained or even further improved for several metrics during the early and main outbreak periods, indicating that the effect of the quality improvement program persists over time.

As expected, stroke admissions in Taiwan decreased by approximately $13 \%$ to $16 \%$ in the main COVID-19 outbreak phase in the main COVID-19 outbreak phase. However, the reduction appears much less than the global average of $42 \%$ reduction reported by the World Stroke Organization. ${ }^{4}$ During the outbreak patients with mild stroke symptoms may be less willing or may took longer time to visit the hospital. ${ }^{5}$ Our data showed a trend of decreasing proportion of mild stroke (National Institutes of Health Stroke Scale [NIHSS] <4; 40.2\% vs. 42.6\%; OR, 0.91; $P=0.07$ ) and mild to moderate stroke (NIHSS $<10 ; 73.3 \%$ vs. 76.0\%; OR, 0.87; $P=0.02$ ) in 2020 compared with 2019. Besides, the number of early arrivals was higher in 2020 than 2019; these patients most likely had considerable neurological signs and were thus sent to hospitals earlier. The proportion of patients receiving acute reperfusion therapy did not decrease in 2020, suggesting that the quality of acute intervention of stroke were still maintained during the pandemic.

When encountering an outbreak of a highly contagious disease, the performance of timely and emergent acute stroke care could be compromised. Modification of the hyperacute stroke management protocol has been advocated during this pandemic in many countries, including Taiwan. ${ }^{6,7}$ In this study, the proportion of patients with a door-to-computed tomography time $\leq 25$ minutes was lower in the main outbreak phase, which would have resulted in delaying hyperacute stroke management. ${ }^{8} \mathrm{Nev}-$ ertheless, the proportion of patients with a door-to-needle time $\leq 60$ minutes in our study was not affected, suggesting that the participating hospitals made their best effort to adhere to hyperacute stroke protocols.

The main limitation of our study was that we were able to use month-based hospital-level data only, and detailed individual patient-level data such as demographic profiles and stroke severities could not be analyzed. In addition, Taiwan was far less severely affected by the pandemic compared with other countries, hence the generalizability of our results should be taken into consideration.

In conclusion, we showed that the collateral adverse effect on stroke admission even in a country less affected by COVID-19. Well-implemented performance improvement program could lead to a fair maintenance of stroke care quality even during the public health crises.

\section{Supplementary materials}

Supplementary materials related to this article can be found online at https://doi.org/10.5853/jos.2020.02292.

\section{References}

1. Schwamm LH, Fonarow GC, Reeves MJ, Pan W, Frankel MR, Smith EE, et al. Get With The Guidelines-Stroke is associated with sustained improvement in care for patients hospitalized with acute stroke or transient ischemic attack. Circulation 2009;119:107-115.

2. Hsieh $\mathrm{Fl}$, Jeng JS, Chern CM, Lee TH, Tang SC, Tsai LK, et al. Quality improvement in acute ischemic stroke care in Taiwan: the breakthrough collaborative in stroke. PLoS One 2016;11: e0160426.

3. Wang CJ, Ng CY, Brook RH. Response to COVID-19 in Taiwan: big data analytics, new technology, and proactive testing. JAMA 2020;323:1341-1342.

4. Liu R, Zhao J, Fisher M. The global impact of COVID-19 on acute stroke care. CNS Neurosci Ther 2020;26:1103-1105.

5. Teo KC, Leung WCY, Wong YK, Liu RKC, Chan AHY, Choi OMY, et al. Delays in stroke onset to hospital arrival time during COVID-19. Stroke 2020;51:2228-2231.

6. Khosravani $H_{1}$ Rajendram $P$, Notario L, Chapman MG, Menon BK. Protected code stroke: hyperacute stroke management during the coronavirus disease 2019 (COVID-19) pandemic. Stroke 2020;51:1891-1895.

7. Lin PY, Chang YM, Huang CY, Lin $\mathrm{CH}$, Chuang MT, Chen PL, et al. Management of hyperacute stroke during the coronavirus disease of 2019 pandemic: the modified code stroke in a medical center in Taiwan. J Stroke 2020;22:278-281.

8. Kerleroux B, Fabacher T, Bricout N, Moïse M, Testud B, Vingadassalom $\mathrm{S}$, et al. Mechanical thrombectomy for acute ischemic stroke amid the COVID-19 outbreak: decreased activity, and increased care delays. Stroke 2020;51:2012-2017. 
Correspondence: Sung-Chun Tang

Department of Neurology, National Taiwan University Hospital, No. 7, ChungShan South Road, Taipei 100, Taiwan

Tel: +886-2-23562144

Fax: +886-2-23418395

E-mail: sctang@ntuh.gov.tw

https://orcid.org/0000-0003-3731-5973

Received: June 12, 2020

Revised: July 31, 2020

Accepted: August 3, 2020

The authors have no financial conflicts of interest. 


\section{Supplementary methods}

\section{Participating hospitals}

The data covered 18 hospitals in Taiwan's different administrative districts; these districts together contain $>65 \%$ of the total population. The enrolled hospitals included seven medical centers and 11 community hospitals, and the corresponding principal investigators were all members of Taiwan Stroke Society (Supplementary Table 1) and participants of the BTSStroke activity, where they received training in the measurement of quality and safety from trained neurologists, study nurses, and stroke case managers. The details of the training and data collection process involved in the BTS-Stroke activity has been reported previously. ${ }^{6}$ Individual patient-level information was de-identified before analysis. This study was approved by the National Taiwan University Hospital Research Ethics Committee, No. 202004035RINA.

\section{Quality metrics for stroke care}

The original BTS-Stroke quality metrics were established in 2010. It included 14 performance measures and safety indicators. These indicators are the percentage of (1) patients presenting with stroke symptoms for $<2$ hours who have a door-to-computed tomography time $\leq 25$ minutes; (2) patients who arrived at the participating hospital $<2$ hours after symptom onset who use an intravenous tissue plasminogen activator (IV-tPA; IV-tPA for early arrival); (3) patients with acute ischemic stroke (AIS) who receive IV-tPA treatment; (4) patients who arrived $<2$ hours after symptom onset who have a of door-to-needle time $\leq 60$ minutes; (5) patients who underwent IV-tPA treatment who developed symptomatic intracerebral hemorrhage (ICH); (6) patients with AIS who receive intraarterial thrombolysis; (7) patients who use antithrombotic medication use $\leq 48$ hours upon admission (early antithrombotic use); (8) patients who undergo dysphagia screening before any oral intake; (9) patients with atrial fibrillation who are prescribed oral anticoagulants at discharge; (10) patients with a lipid-lowering drug prescription for low-density lipoprotein $\geq 100 \mathrm{mg} / \mathrm{dL}$ at discharge (lipid-lowering drug use); (11) patients with an antithrombotic prescription at discharge (antithrombotic use at discharge); (12) patients who are evaluated for stroke rehabilitation services (rehabilitation evaluation); (13) patients (and/or caregivers) who undergo stroke education (stroke education); and (14) patients with stroke who have a 30-day mortality. Since 2015, endovascular thrombectomy (EVT) has become the standard treatment for patients with AIS with large vessel occlusion. Therefore, we replaced intraarterial thrombolysis with EVT and added metric 15: symptomatic ICH after EVT (Supplementary Table 2). Stroke severity, represent- ing by National Institutes of Health Stroke Scale (NIHSS), was not included in the BTS-Stroke activity. Nevertheless, we collected patients' NIHSS score according to four strata as $<4,4$ to 10 , 11 to 20 , and $>20$.

All quality metrics were reviewed on a monthly basis in each participating hospital. Furthermore, the total number of monthly stroke admissions (including those of AIS, transient ischemic attack and hemorrhagic stroke) were recorded. Since the BTSStroke activity mainly focused on the AIS-related quality metrics, we further recorded number of AIS admission. The study period was January 1 to March 31,2020, and the control period was January 1 to March 31, 2019.

\section{COVID-19 statistics}

Coronavirus disease 2019 (COVID-19) statistics were collected from the bulletins and press releases of the Central Epidemic Command Center (CECC), a specialized task force under Taiwan's Centers for Disease Control. We collected the daily numbers of confirmed cases (reported from home quarantine and enhanced surveillance).

\section{Statistical analysis}

The mean daily stroke and AIS admissions were calculated from data on their monthly counterparts (monthly figures/number of days of the month). The changes over the 3 consecutive months were estimated using a generalized estimating equation. The difference in the mean values between different study periods were compared using Poisson regression and expressed as incidence rate ratios and their 95\% confidence intervals.

All quality metrics were represented as percentages (\%). Because the denominator in these percentages may change according to which patients are covered by any given quality metrics, both the numerator and denominator are reported in the results. When appropriate, the chi-square test or Fisher's exact test was used to compare quality metrics between the study and control periods. Logistic regression analysis was used to calculate the odds ratios for quality of care between the 2 periods, and the penalized maximum likelihood (Firth method) was used for parameter estimation to determine the likelihood of rare outcomes. ${ }^{\text {? }}$

In brief, we first compared the first quarters of 2019 and 2020 with respect to the mean numbers of daily stroke admission of stroke and the quality-metric percentages. Prespecified subgroup analyses were performed for medical centers and community hospitals. Because the number of confirmed COVID-19 cases increased substantially in mid-March, we considered March 2020 as the most affected month (i.e., the main outbreak phase). Thus, we further compared data for March 
2020 with those of January to February 2020 (i.e., the early outbreak phase) and the first quarter of 2019 (i.e., the control phase), respectively. All statistical analyses were performed using SAS version 9.4 (SAS Institute Inc., Cary, NC, USA). 
Supplementary Table 1. List of the participating hospitals and team members of the Taiwan Stroke Society Investigators

\begin{tabular}{|c|c|c|c|}
\hline Hospital & Accredited type & Districts & Team members \\
\hline National Taiwan University Hospital & Medical center & Taipei city & $\begin{array}{l}\text { Chih-Hao Chen, MD, PhD; Sung-Chun Tang, MD, PhD; } \\
\text { Jiann-Shing Jeng, MD, PhD }\end{array}$ \\
\hline Taipei Veterans General Hospital & Medical center & Taipei city & Nai-Fang Chi, MD; I-Hui Lee, MD, PhD \\
\hline Tri-Service General Hospital & Medical center & Taipei city & Chung-Hsing Chou, MD, PhD; Jiunn-Tay Lee, MD \\
\hline Mackay Memorial Hospital & Medical center & Taipei city & Ya-Ju Lin, MD, MSc; Helen L Po, MD \\
\hline Shin Kong Wu Ho-Su Memorial Hospital & Medical center & Taipei city & Hsu-Ling Yeh, MD; Li-Ming Lien, MD, PhD \\
\hline Linkou Chang Gung Memorial Hospital & Medical center & Taoyuan city & $\begin{array}{l}\text { Chi-Hung Liu, MD, MSc; Chien-Hung Chang, MD; } \\
\text { Tsong-Hai Lee, MD, PhD }\end{array}$ \\
\hline National Cheng Kung University Hospital & Medical center & Tainan city & Yu-Ming Chang, MD; Pi-Shan Sung, MD \\
\hline Keelung Chang Gung Memorial Hospital & Community hospital & Keelung city & Feng-Chieh Su, MD; Wen-Yi Huang, MD, PhD \\
\hline Taipei Medical University Shuang-Ho Hospital & Community hospital & New Taipei city & Lung Chan, MD, PhD; Chaur-Jong Hu, MD, PhD \\
\hline Taipei Tzu Chi Hospital & Community hospital & New Taipei city & Po-Jen Hsu, MD; Shinn-Kuang Lin, MD \\
\hline Cardinal Tien Hospital & Community hospital & New Taipei city & Chung-Fen Tsai, MD, PhD; Ping-Keung Yip, MD \\
\hline En Chu Kong Hospital & Community hospital & New Taipei city & Hai-Jui Chu, MD; Yu Sun, MD, PhD \\
\hline Landseed International Hospital & Community hospital & Taoyuan city & Yu-Wei Chen, MD, PhD; Chi-Jen Chen, MD \\
\hline National Taiwan University Hospital Hsin-Chu Branch & Community hospital & Hsinchu city & Hui-Chi Yang, BS; Kai-Hsiang Chen, MD \\
\hline National Taiwan University Hospital Yunlin Branch & Community hospital & Yunlin county & Yiman Sun, BS; Kai-Chieh Chang, MD \\
\hline Chiayi Chang Gung Memorial Hospital & Community hospital & Chiayi county & Chun-Hsien Lin, MD; Yen-Chu Huang, MD \\
\hline Ditmanson Medical Foundation Chiayi Christian Hospital & Community hospital & Chiayi city & Yu-Hsiang Su, MD; Sheng-Feng Sung, MD, MS \\
\hline E-Da Hospital & Community hospital & Kaohsiung city & Meng-Tsang Hsieh, MD; Tzu-Tung Tsai, MD \\
\hline
\end{tabular}


Supplementary Table 2. List of the quality metrics of stroke care

\begin{tabular}{|c|c|c|}
\hline Quality metrics & Denominator & Numerator \\
\hline Door-to-CT $\leq 25 \mathrm{~min}$ & $\begin{array}{l}\text { Patients who arrived at a participating hospital }<2 \mathrm{hr} \text { from } \\
\text { symptom onset }\end{array}$ & $\begin{array}{l}\text { Patients in the denominator who received head CT } \leq 25 \\
\text { min of hospital arrival }\end{array}$ \\
\hline IV-tPA for early arrival & $\begin{array}{l}\text { Patients who arrived at a participating hospital }<2 \mathrm{hr} \text { from } \\
\text { symptom onset, and fulfilled the indication for IV-tPA }\end{array}$ & Patients in the denominator who received IV-tPA \\
\hline IV-tPA percentage & AIS patients admitted in the participating hospitals & Patients in the denominator who received IV-tPA \\
\hline Door-to-needle $\leq 60 \mathrm{~min}$ & Patients who received IV-tPA & $\begin{array}{l}\text { Patients in the denominator who received IV-tPA } \leq 60 \mathrm{~min} \\
\text { of hospital arrival }\end{array}$ \\
\hline Symptomatic ICH after IV-tPA & Patients who received IV-tPA & Patients in the denominator who have symptomatic ICH \\
\hline EVT percentage & AIS patients admitted in the participating hospitals & Patients in the denominator who received EVT \\
\hline Symptomatic ICH after EVT & Patients who received EVT & Patients in the denominator who have symptomatic ICH \\
\hline Dysphagia screening & AIS patients admitted in the participating hospitals & $\begin{array}{l}\text { Patients in the denominator who received dysphagia } \\
\text { screening before their first oral intake }\end{array}$ \\
\hline Early antithrombotics use & AIS patients admitted in the participating hospitals & $\begin{array}{l}\text { Patients in the denominator who received any } \\
\text { antithrombotic agent (antiplatelets or anticoagulants) } \\
\text { within } 48 \mathrm{hr} \text { of hospital arrival }\end{array}$ \\
\hline Anticoagulants for $\mathrm{AF}$ & $\begin{array}{l}\text { AIS patients who have } \mathrm{AF} \text { and are indicated for } \\
\text { anticoagulation }\end{array}$ & $\begin{array}{l}\text { Patients in the denominator who received } \\
\text { anticoagulant }\end{array}$ \\
\hline Lipid-lowering drugs use & $\begin{array}{l}\text { AIS patients admitted in the participating hospitals who } \\
\text { have } \mathrm{LDL}>100 \mathrm{mg} / \mathrm{dL}\end{array}$ & $\begin{array}{l}\text { Patients in the denominator who received any } \\
\text { lipid-lowering agent at discharge }\end{array}$ \\
\hline Antithrombotics use at discharge & AIS patients admitted in the participating hospitals & $\begin{array}{l}\text { Patients in the denominator who received any } \\
\text { antithrombotic agent (antiplatelets or anticoagulants) at } \\
\text { discharge }\end{array}$ \\
\hline Rehabilitation evaluation & AIS patients admitted in the participating hospitals & $\begin{array}{l}\text { Patients in the denominator who received any } \\
\text { rehabilitation evaluation }\end{array}$ \\
\hline Stroke education & AIS patients admitted in the participating hospitals & $\begin{array}{l}\text { Patients in the denominator who received any stroke } \\
\text { education }\end{array}$ \\
\hline 30-day mortality & AIS patients admitted in the participating hospitals & $\begin{array}{l}\text { Patients in the denominator who died within } 30 \text { day of } \\
\text { hospital arrival }\end{array}$ \\
\hline
\end{tabular}

$\mathrm{CT}$, computed tomography; IV-tPA, intravenous tissue plasminogen activator; ICH, intracerebral hemorrhage; EVT, endovascular thrombectomy; AIS, acute ischemic stroke; $A F$, atrial fibrillation; LDL, low-density lipoprotein. 